\title{
FATORES ASSOCIADOS AO TRATAMENTO DE ADOLESCENTES COM TRANSTORNO DE PERSONALIDADE BORDERLINE REVISÃO INTEGRATIVA
}

\author{
FACTORS ASSOCIATED WITH TREATMENT OF ADOLESCENTS WITH \\ BORDERLINE PERSONALITY DISORDER: AN INTEGRATIVE REVIEW
}

\author{
Gerusa Marcondes Pimentel De Abreu Lima, Claudinei José Gomes Campos
}

Universidade Estadual de Campinas - Unicamp

\begin{abstract}
The Borderline Personality Disorder is an instability pattern in interpersonal relationships, self-image, emotions and impulsivity. The aim of this study was to identify in the literature, the role of parents and their commitment to exercise significant interventions on the treatment carried out with adolescents, considering also the specialized treatment received by their children. Method: this study is an integrative review of scientific literature included in the period 20102015, in indexing sources LILACS; PUBMED, MEDLINE; BIREME; IBECS; COCHRANE and $S C I E L O$. The guiding question for the review was: what are the associated factors with parents in the treatment of adolescents with Borderline Personality Disorder and what are the interventions received by children for treatment? The content analysis was performed for the emergence of the themes. Results: There were 11 articles suitable for assessing. It was observed that actions should be encouraged to expand the procedures to support the family in the psychotherapeutic process. It is suggested that family therapy may act as a protective way for the pathology involved. Conclusion: The developed affective bonds can be considered indispensable factors for the treatment abandonment not occur, or even that this treatment can be followed with no interruption.
\end{abstract}

Key words: Borderline personality disorder, treatment, adolescent, family
Resumo

O Transtorno de Personalidade Borderline faz referência a um padrão de instabilidade nas relações interpessoais, autoimagem e afetos além da impulsividade. O Objetivo desse estudo foi identificar na literatura o papel dos genitores e seu comprometimento ao exercerem intervenções significativas diante do tratamento realizado com adolescentes, considerando também, o tratamento especializado recebidas por seus filhos. Método: revisão integrativa de literatura científica compreendidas no período de 2010 a 2015, nas fontes indexadoras, LILACS; PUBMED;MEDLINE; BIREME;IBECS; COCHRANE $e$ a SCIELO. A pergunta norteadora para a revisão foi: quais são os fatores associados aos pais no tratamento de adolescentes com Transtorno de Personalidade Borderline e quais são as intervenções recebidas pelos filhos para 0 tratamento? Foi realizada a análise de conteúdo, para a emersão das categorias temáticas. Resultados: Foram considerados aptos para a apreciação 11 artigos. Evidenciou-se que devem ser incentivadas, ações que propendam a ampliar os procedimentos que apoiem a família no processo psicoterapêutico. Sugere-se que a terapia familiar possa agir de forma protetiva em torno da patologia envolvida. Conclusão: os vínculos afetivos desenvolvidos podem ser considerados fatores indispensáveis para que não aconteça o abandono ao tratamento, ou mesmo, que esse possa ser seguido de forma não interrupta.

Palavras chave: Transtorno de personalidade borderline, tratamento, adolescente, familia 


\section{Introdução}

O Transtorno de Personalidade Borderline (TPB) é classificado nosograficamente como um transtorno de personalidade e o termo Borderline usado de forma ampla, para referir-se a uma estrutura de organização de personalidade ou determinando um espectro. O TPB pode ser conceituado, como padrão de instabilidade nas relações interpessoais, na autoimagem e nos afetos, além da impulsividade ${ }^{1}$.

Para a clarificação dos conceitos, o TPB está inserido na Seção II do grupo B do Manual Diagnóstico e Estático dos Transtornos Mentais (DSM-5). A prevalência do transtorno na população geral é estimada em 1,6\%, porém, esse número pode subir para $5,9 \%$. A prevalência do TPB nas unidades de atenção primária abrange cerca de $6 \%$ e essa porcentagem aumenta para $10 \%$ entre os indivíduos observados em ambulatório mentais e unidades de saúde, elevando para cerca de $20 \%$ entre os pacientes psiquiátricos internados ${ }^{1}$.

Os indivíduos diagnosticados com TPB vivenciam seus sintomas, evidentemente, dentro de um contexto fundamentado em crenças culturais, valores e normas de conduta $^{2,}{ }^{3}$. Comumente, são pacientes que não aderem ao tratamento ou recusam os procedimentos clínicos e exames laboratoriais; não comparecendo às sessões psicológicas adequadamente, além de fazer uso inapropriado dos medicamentos prescritos e, consequentemente, da automedicação ${ }^{4}$. Desse modo, são consideradas pessoas inconvenientes, que geralmente fazem uso dos atendimentos de emergência da psiquiatria e, de outras clínicas médicas, devido as suas condutas agressivas e autodestrutivas $^{5}$.

Sabe-se, que o TPB, igualmente em adultos, pode ser identificado clinicamente, com os sintomas evidenciados nos adolescentes. O púbere tem em sua convivência, relações muitas vezes problemáticas, como por exemplo: a própria organização e construção da sua identidade, o que pode ocasionar abalos em meio à procura da dependência e a necessidade de constituir sua individuação ${ }^{6}$.

Estudos retratam que o adolescente com TPB geralmente são provenientes de famílias marcadas por altos níveis de psicopatologia, bem como estilos parentais disfuncionais que tendem a gerar atitudes negativas contra os pais ${ }^{7}$. A relação diádica entre pai e filho, foi estudada com o objetivo de analisar a qualidade dessa interação, a fim de associar sintomas encontrados no TPB em adolescentes que poderiam indicar algum comportamento para o ato suicida ou auto lesão ${ }^{8}$.

Por outro lado, há pesquisas retratando que entre outras doenças mentais graves, O TPB causa impacto à saúde mental do cuidador responsável pelo adolescente acometido pela doença. Nesse sentido, pode ocasionar para algum comprometimento de adoecimento psíquico, visto que se sentem sobrecarregados, demonstrando, humores inconsistentes, quadros de ansiedade e transtorno de estresse póstraumático ${ }^{9}$. Conclui-se que o transtorno afeta gravemente a vida do individuo, pois, ocasiona danos expressivos a si próprios como àqueles que fazem parte do seu circulo social ${ }^{4}$.

Perante tal afirmação sobre a consideração descrita, a questão torna- se peculiar quando apontado à importância do tratamento farmacológico em pacientes com TPB. É notório que à psicofarmacoterapia aplicada, melhora o restabelecimento do funcionamento psicossocial em adolescentes com transtorno de personalidade borderline levando-a uma intervenção qualitativa em suas relações sociais $^{10}$

Diante disso, o objetivo desse estudo foi identificar na literatura vigente os aspectos psicossociais, parentais e o tratamento realizado com adolescentes com diagnóstico de transtorno de personalidade Borderline, a fim de proporcionar dados e esclarecimentos atuais sobre o agravante adoecimento psíquico.

\section{Metodologia}

Para a realização desse estudo foi eleita à metodologia de revisão integrativa que incide em uma apreciação e análise extensa da literatura, que agrupa inúmeras publicações, com o objetivo primordial de responder a uma determinada indagação, com a finalidade de buscar a compreensão profunda daquilo que está sendo investigado e assim, prover novas discussões dos resultados encontrados ${ }^{11}$.

Há algumas etapas fundamentais a ser seguido na construção da revisão integrativa de literatura, dentre elas, o tema central da pesquisa e seu objetivo; elaboração da pergunta da pesquisa; categorização dos critérios de exclusão e inclusão para a escolha da amostra e investigação na literatura, demarcação das informações a serem estudadas dos manuscritos elegidos; análise dos artigos e por fim, a intepretação e apresentação da revisão 
integrativa ${ }^{12,13}$. A pergunta norteadora para a revisão foi: "qual o papel dos pais quando associados ao tratamento de adolescentes com Transtorno de Personalidade Borderline e quais são as intervenções recebidas pelos filhos para o tratamento?".

Para a demarcação da amostra, foram constituídos os seguintes critérios de inclusão: artigos originais publicados nos idiomas inglês, português e espanhol compreendido entre os anos de 2010 até agosto de 2015, desde que o tema respondesse a questão norteadora, ou seja, os artigos necessitariam abranger o tema discutido: "abordar o envolvimento dos pais no tratamento do filho adolescente com TPB" e que estivessem disponibilizados nas seguintes bases de dados: Literatura Latino-Americana e do Caribe em Ciências da Saúde (LILACS); Literatura Internacional em Ciências da Saúde (PUBMED/MEDLINE); BIREME; IBECS; a Biblioteca COCHRANE e a SCIELO. Os termos utilizados como palavras chaves, tanto na língua portuguesa como na inglesa foram identificadas nos descritores em Ciências da Saúde (DECs) e Medicais Subject Headings (MESH): tratamento, adolescente, transtorno de personalidade Borderline, família, relacionando-os por meio do operador boleano "AND". Quanto aos critérios de exclusão, foram eliminadas, as dissertações, teses e artigos de revisões integrativas, e artigos que não mencionavam os pais ou a família nos resultados de sua pesquisa.

O seguinte passo para a inclusão das pesquisas foi à realização de leitura exaustiva dos resumos e títulos de cada um dos artigos encontrados pelos autores de forma autônoma, para averiguar a relação com o objetivo do presente estudo, ou seja, se o artigo encontrado respondia a indagação.

\section{Resultados e Discussão}

Obteve-se, após a busca nas bases de dados o total de 991 artigos. O que pode ser verificado conforme a Tabela 1, na qual são identificadas as bases de dados.

Tabela 1. Distribuição dos artigos encontrados, excluídos e selecionados nas bases eletrônicas de dados entre 2010

\begin{tabular}{|c|c|c|c|c|c|}
\hline & & & & & \\
\hline Dados & Encontrados & Excluídos & $\begin{array}{c}\text { Amostra } \\
\text { parcial }\end{array}$ & $\begin{array}{c}\text { Excluídos da } \\
\text { amostra } \\
\text { parcial }\end{array}$ & $\begin{array}{c}\text { Amostra } \\
\text { Final }\end{array}$ \\
\hline PUBMED & 730 & 598 & 132 & 98 & 6 \\
\hline LILACS & 4 & 3 & 1 & 0 & 0 \\
\hline IBECS & 4 & 3 & 1 & 0 & 0 \\
\hline MEDLINE & 151 & 112 & 39 & 34 & 5 \\
\hline COCHRANE & 102 & 95 & 7 & 7 & 0 \\
\hline Total & 991 & 811 & 180 & 139 & 11 \\
\hline
\end{tabular}

Posteriormente a efetivação da leitura do título e do resumo, visto que havia textos que não se encontravam disponíveis na íntegra, e, além disso, o resumo não forneceu dados suficientes para a realização da análise com fidedignidade e, principalmente, por não responder a pergunta que norteava a busca dos artigos, por isso, foram excluídos. Da mesma, foram removidos os artigos que estavam em duplicidade. Portanto, Os artigos excluídos da amostra parcial indicavam 64 manuscritos em duplicidade com outras fontes indexadores. Além disso, artigos que não abordavam o objetivo do trabalho proposto, e não possuíam em seus resultados os apontamentos do papel ou função dos pais no tratamento, apesar de conter a citação dos seus descritores, não era questionado o tema principal da presente revisão integrativo.

Dessa forma, foram excluídas em sua totalidade 980 publicações conforme exemplificada na Tabela 1. Sendo apenas 11 
artigos considerados aptos para a apreciação. Verificamos que os descritores utilizados nos forneceram um grande número de artigos indexados na base de dados, porém, ao final da amostra, obtivemos um número relativamente abaixo do esperado. Observou-se que inicialmente, ocorreu alta concentração de artigos no banco de dados da PUBMED, encontrados 730 manuscritos utilizando-se, os descritores de busca já citados. Porém, ao analisar com profundidade os artigos, a amostra final foi composta de apenas seis artigos. Abaixo o Quadro 1. Com as descrições das pesquisas selecionadas, com destaque para: os autores, ano de publicação, e o fator de impacto dos periódicos.

Em relação ao país de origem dos autores dos artigos acima citados, ficou assim compilado: um da Croácia; dois sendo multinacionais: Reino Unido e Estados Unidos e Itália com Alemanha; dois do Canadá; um da China e cinco dos Estados Unidos; todos tiveram seus trabalhos publicados em periódicos de grande impacto. Dentre os artigos selecionados, considerando a titulação dos primeiros autores, seis foram publicados por psicólogos clínicos $14,15,16,17,18,19,20$ e quatro por psiquiatras $^{5,21,22,23}$, importante considerar que houve a interação interdisciplinar entre seus autores. Corroborando que os profissionais da área da saúde mental (psicologia e psiquiatria) evidência um maior interesse em publicar assuntos relacionados ao tratamento do adolescente com o TPB, observando que em todos os manuscritos encontrados relataram a importância ou nomearam a família no tratamento realizado com os adolescentes.

Outro ponto em destaque da relação dos artigos constituídos para tal avaliação é o seu alto fator de impacto (FI) nos periódicos analisados, variando entre 1,3 A 4,86, oferecendo ao leitor a credibilidade e qualidade dos estudos aferidos. Visto que, o fator de impacto representa os números médios, referentes às citações dos manuscritos científicos e, concomitantemente, esses dados são concebíveis para analisar a relevância e o destaque do periódico.

Quadro 1. Relação dos artigos nos bancos de dados para a revisão integrativa

\begin{tabular}{|c|c|c|c|c|c|}
\hline №. & AUTOR & $\begin{array}{l}\text { ANO/ Fator } \\
\text { de impacto } \\
\text { do periódico } \\
\text { revista/ } \\
\text { INDEXAÇÃO }\end{array}$ & REVISTA & ARTIGO & RESULTADOS \\
\hline 1 & $\begin{array}{l}\text { Marcinko D, } \\
\text { Bilic V. }\end{array}$ & $\begin{array}{l}2010 \\
\mathrm{Fl}=1,3 \\
\text { Medline }\end{array}$ & $\begin{array}{l}\text { Psychiatr } \\
\text { Danub;22(2): } \\
\text { 257-60, 2010 } \\
\text { Jun }\end{array}$ & $\begin{array}{l}\text { Family therapy as } \\
\text { addition to } \\
\text { individual therapy } \\
\text { and } \\
\text { psychopharmacoth } \\
\text { erapy in late } \\
\text { adolescent female } \\
\text { patients suffering } \\
\text { from borderline } \\
\text { personality } \\
\text { disorder with } \\
\begin{array}{l}\text { comorbidity and } \\
\text { positive suicidal } \\
\text { history }\end{array}\end{array}$ & $\begin{array}{l}\text { Enfatizam a importância e a eficácia do } \\
\text { tratamento psicoterapêutico, } \\
\text { sobretudo envolvendo a família/pais na } \\
\text { intervenção do tratamento com os } \\
\text { adolescentes borderlaines. }\end{array}$ \\
\hline 2 & $\begin{array}{l}\text { Winsper } \\
\text { C, Zanarini } \\
\text { M, Wolke D. }\end{array}$ & $\begin{array}{l}2012 \\
\mathrm{Fl}=2,467 \\
\text { PUBMED }\end{array}$ & $\begin{array}{l}\text { Psychiatry } \\
\text { Research. } 201 \\
2 \\
\text { Nov; } 42(11): 24 \\
05-20 . .\end{array}$ & $\begin{array}{l}\text { Prospective study } \\
\text { of family adversity } \\
\text { and malada ptive } \\
\text { parenting in } \\
\text { childhood } \\
\text { and borderline pers } \\
\text { onality dis order } \\
\text { symptoms in a non- } \\
\text { clinical population } \\
\text { at } 11 \text { years. }\end{array}$ & $\begin{array}{l}\text { Crianças que em sua infância tiveram } \\
\text { relações conflituosas com seus pais, } \\
\text { estão em maior risco de } \\
\text { desenvolverem sintomas para o TPB } \\
\text { aos } 11 \text { anos. }\end{array}$ \\
\hline 3 & Larrivée MP. & $\begin{array}{l}2013 \\
\mathrm{Fl}=1,97 \\
\text { Medline }\end{array}$ & $\begin{array}{l}\text { Dialogues Clin } \\
\text { Neurosci; } \\
\text { 15(2): } 171-9 \text {, } \\
2013 \text { Jun. }\end{array}$ & $\begin{array}{l}\text { Borderline } \\
\text { personality } \\
\text { disorder } \\
\text { adolescente: in } \\
\text { He-Who-must-not- } \\
\text { be-named of } \\
\text { psychiatri }\end{array}$ & $\begin{array}{l}\text { O sofrimento vivenciado pela família } \\
\text { com o filho com TPB tem que ser } \\
\text { validado. }\end{array}$ \\
\hline
\end{tabular}




\begin{tabular}{|c|c|c|c|c|c|}
\hline 4 & $\begin{array}{l}\text { Wang } Y \text {, } \\
\text { X, Cai Lu } \\
\text { Wang } \quad Q \text {, } \\
\text { Wang } M, \text { Yi J, } \\
\text { Yao S. }\end{array}$ & $\begin{array}{l}2013 \\
\mathrm{Fl}=2,21 \\
\text { Medline }\end{array}$ & $\begin{array}{l}\text { BMC } \\
\text { Psychiatry,200 } \\
9 ; 13: 116\end{array}$ & $\begin{array}{l}\text { Screening cluster A } \\
\text { and cluster B } \\
\text { personality } \\
\text { disorders in } \\
\text { Chinese high school } \\
\text { students. }\end{array}$ & $\begin{array}{l}\text { O TPB durante a adolescência poderá } \\
\text { aumentar o risco para } \\
\text { comportamentos agressivos e até } \\
\text { mesmo para o ato suicída. Além disso, } \\
\text { o comprometimento da estrutura } \\
\text { familiar poderá afetar } \\
\text { desenvolvimento de TPB. }\end{array}$ \\
\hline 5 & $\begin{array}{l}\text { Whalen } \\
\text { DJ, Scott } \\
\text { LN, Jakubows } \\
\text { ki } \\
\text { KP, McMakin } \\
\text { DL, Hipwell } \\
\text { AE, Silk } \\
\text { JS, Stepp SD. }\end{array}$ & $\begin{array}{l}2014 \\
\mathrm{Fl}=3,221 \\
\text { Medline }\end{array}$ & $\begin{array}{l}\text { Personality } \\
\text { Disorders: } \\
\text { Theory, } \\
\text { Research, and } \\
\text { Treatment, Vol } \\
\text { 5(1), Jan 2014, } \\
\text { 88-96. }\end{array}$ & $\begin{array}{l}\text { Affective behavior } \\
\text { during mother- } \\
\text { daughter conflict } \\
\text { and borderline } \\
\text { personality } \\
\text { disorder severity } \\
\text { across } \\
\text { adolescence. }\end{array}$ & $\begin{array}{l}\text { As relações saudaveis e positivas } \\
\text { desenvolvidas entre mães e filha, pode } \\
\text { ser um fator protetor importante na } \\
\text { determinação e da gravidade do TPB } \\
\text { durante a adolescência, além disso, os } \\
\text { esforços associados a intervenção } \\
\text { estãopresentes. }\end{array}$ \\
\hline 6 & $\begin{array}{l}\text { Kim,S, Sharp } \\
\text { C, C C. }\end{array}$ & $\begin{array}{l}2014 \\
\mathrm{Fl}=3.221 \\
\text { PUBMED }\end{array}$ & $\begin{array}{l}\text { Personality } \\
\text { Disorders: } \\
\text { Theory, } \\
\text { Research, and } \\
\text { Treatment, Vol } \\
\text { 5(2), Apr 2014, } \\
\text { 125-136 }\end{array}$ & $\begin{array}{l}\text { The protective role } \\
\text { of attachment } \\
\text { security for } \\
\text { adolescent } \\
\text { borderline } \\
\text { personality } \\
\text { disorder features } \\
\text { via enhanced } \\
\text { positive emotion } \\
\text { regulation } \\
\text { strategies. }\end{array}$ & $\begin{array}{l}\text { O Apego seguro desenvolvidos entre os } \\
\text { adolescentes e seus pais, são fatores } \\
\text { contra o aparecimento do TPB em seus } \\
\text { filhos. Ou seja, reforçam estategias } \\
\text { positivas na regulação emocional em } \\
\text { relação as intervenções no } \\
\text { desenvolvimento do TPB. }\end{array}$ \\
\hline 7 & $\begin{array}{l}\text { Nelson } \\
\text { DA, Coyne } \\
\text { SM, Swanson } \\
\text { SM, Hart } \\
\text { CH, Olsen JA. }\end{array}$ & $\begin{array}{l}2014 \\
\text { FI-4.891 } \\
\text { PUBMED }\end{array}$ & $\begin{array}{l}\text { Development } \\
\text { and } \\
\text { Psychopatholo } \\
\text { gy_2014 } \\
\text { Aug;26(3):773- } \\
87 .\end{array}$ & $\begin{array}{l}\text { Parenting, } \\
\text { relational } \\
\text { aggression, } \\
\text { and borderline pers } \\
\text { onality features: } \\
\text { associations over } \\
\text { time in a Russian } \\
\text { longitudinal } \\
\text { sample. }\end{array}$ & $\begin{array}{l}\text { Os resultados mostram que os fatores } \\
\text { parentais são } \\
\text { igualmente importante para a } \\
\text { compreensão do desenvolvimento } \\
\text { tanto do TPB como do comportamento } \\
\text { agressivo. Os resultados } \\
\text { tem implicações importantes para o } \\
\text { tratamento do TPB. Os pais têm o } \\
\text { poder de mudar a sua forma de relação } \\
\text { afetiva para auxiliar no tratatamento e } \\
\text { auxiliar na construção de uma relação } \\
\text { vincular positiva, sendo um fator } \\
\text { preditivo para o não aparecimento dos } \\
\text { sintomas do TPB.. }\end{array}$ \\
\hline 8 & $\begin{array}{l}\text { Desrosiers L, } \\
\text { Saint-Jean M, } \\
\text { Breton JJ }\end{array}$ & $\begin{array}{l}2015 \\
\mathrm{FI}=1.441 \\
\text { PUBMED }\end{array}$ & $\begin{array}{l}\text { Psychology } \\
\text { and } \\
\text { psychothera py } \\
88(2): 178-96 \text {, } \\
2015 \text { Jun. }\end{array}$ & $\begin{array}{lr}\text { Treatment } \\
\text { planning: A key } \\
\text { milestone } \\
\text { prevent treatment } \\
\text { dropout } \\
\text { adolescents } \\
\text { borderline } \\
\text { personality } \\
\text { disorder. }\end{array}$ & $\begin{array}{l}\text { Consideram a importancia de avalair } \\
\text { sistemáticamente as causas vulneraveis } \\
\text { associadas ao abandono do } \\
\text { tratamento, antes mesmo da } \\
\text { realização de planos de intervenções, } \\
\text { visto que reduziria o abandono ao } \\
\text { trtamento. }\end{array}$ \\
\hline 9 & $\begin{array}{l}\text { Santisteban } \\
\text { DA, Mena } \\
\text { MP, Muir J, } \\
\text { McCabe BE, } \\
\text { Abalo C, } \\
\text { Cummings } \\
\text { AM. }\end{array}$ & $\begin{array}{l}2015 \\
\mathrm{Fl}=1.169 \\
\text { Medline }\end{array}$ & $\begin{array}{l}\text { Psychiatric } \\
\text { Rehabilitation } \\
\text { Journal, Vol } \\
\text { 38(1), Mar } \\
2015,55-64 .\end{array}$ & $\begin{array}{l}\text { The efficacy of two } \\
\text { adolescent } \\
\text { substance abuse } \\
\text { treatments and the } \\
\text { impact of comorbid } \\
\text { depression: Results } \\
\text { of a small } \\
\text { randomized } \\
\text { controlled trial. }\end{array}$ & $\begin{array}{l}\text { Os resultados destacam as } \\
\text { necessidades do tratamento intensivo, } \\
\text { principalmente com jovens envolvidos } \\
\text { com a justiça que fazem uso de } \\
\text { substancias psicoativas, com TPB e } \\
\text { depresão, junto com orientação } \\
\text { envolvendo a família. }\end{array}$ \\
\hline
\end{tabular}




\begin{tabular}{|c|c|c|c|c|c|}
\hline 10 & $\begin{array}{l}\text { Infurna } \\
\text { MR, Brunner } \\
\text { R, Holz } \\
\text { B, Parzer } \\
\text { P, Giannone } \\
\text { F, Reichl } \\
\text { C, Fischer } \\
\text { G, Resch } \\
\text { F, Kaess M }\end{array}$ & $\begin{array}{l}2015 \\
\mathrm{FI}=2.619 \\
\text { PUBMED }\end{array}$ & $\begin{array}{l}\text { Journal of } \\
\text { personality } \\
\text { disorders. } 201 \\
5 \text { Apr 23:1-16. }\end{array}$ & $\begin{array}{l}\text { The Specific Role of } \\
\text { Childhood Abuse, } \\
\text { Parental Bonding, } \\
\text { and Family Functio } \\
\text { ning in Female } \\
\text { Adolescents } \\
\text { WithBorderline Per } \\
\text { sonality Disorder }\end{array}$ & $\begin{array}{l}\text { Os resultados implicam em promover o } \\
\text { conhecimento (emocional e biologico) } \\
\text { das fases da infancia e adolescencia, } \\
\text { pois, tais conhecimentos geram } \\
\text { prevenção e intervenção adequada } \\
\text { frentes ao problemas e necessidades } \\
\text { surgidas. E as estrategias devem } \\
\text { envolver a família. }\end{array}$ \\
\hline 11 & $\begin{array}{l}\text { Hallquist } \\
\text { MN, Hipwell } \\
\text { AE, Stepp SD. }\end{array}$ & $\begin{array}{l}2015 \\
\mathrm{FI}=4,86 \\
\text { PUBMED }\end{array}$ & $\begin{array}{l}\text { Journal of } \\
\text { Abnormal } \\
\text { Psychology: } 20 \\
15 \\
\text { Aug;124(3):54 } \\
9-64 .\end{array}$ & $\begin{array}{l}\text { Poor self-control } \\
\text { and harsh } \\
\text { punishment in } \\
\text { childhood } \\
\text { prospectively } \\
\text { predict borderline } \\
\text { personality } \\
\text { symptoms in } \\
\text { adolescent girls }\end{array}$ & $\begin{array}{l}\text { O tratamento realizado com o } \\
\text { adolescente com TPB, também é } \\
\text { indicado para os pais, a fim de } \\
\text { promover a interrompção dos sintomas } \\
\text { manifestados. }\end{array}$ \\
\hline
\end{tabular}

Os dados encontrados foram discutidos pela análise de conteúdo $^{24}$ e, posteriormente, articulados dentro das dimensões de unidades de significados: tratamento e relações parentais. Surgindo assim, uma grande categoria, que contemplaria essas dimensões subjetivas analisadas em grande profundidade.

Reflexões dos aspectos sociais e parentais associados aos adolescentes com TPB

Marcinko e Bilic ${ }^{21}$ fazem referência à forma de tratamento que vem sendo realizada com os adolescentes do sexo feminino pondera que os médicos psiquiatras usam de forma excessiva medicamentos farmacológicos, deixando de aconselhar ou indicar a psicoterapia, sendo constatada a sua eficácia nesse tipo de transtorno. Além da medicação e do tratamento ambulatorial, constatou-se que os pais devem ser instruídos sobre a etiologia do transtorno, assim como, a forma como será administrada a profilaxia. No entanto, deve ser incentivadas, ações que propendam a ampliar os procedimentos que apoiem a família no processo psicoterapêutico ${ }^{22}$.

Da mesma forma, baseado em outra pesquisa foi sugerido intervenções para o tratamento clínico do transtorno, como o acompanhamento sistemático para a terapia individual e familiar, como mecanismo, para melhoraria da relação interpessoal ${ }^{15}$. Para tanto, um estudo realizado no Canadá, no ano de 2015 teve como objetivo, verificar os processos envolvidos no abandono de tratamento em adolescente com TPB, considerando três eixos principais: o próprio adolescente, o pai e a configuração do cuidado. Os Resultados apontaram que as características psicológicas, a percepção da doença e os cuidados da saúde mental, além da forma da busca pelo tratamento são fatores essenciais para a vulnerabilidade de abandono de tratamento. Verificou-se que o abandono ao tratamento se tornou um problema quando associado ao aos cuidados, como a própria acessibilidade aos serviços prestados, bem como, a atenção dos médicos para com o paciente, assim à pesquisa revela a necessidade de um planejamento adequado e consistente para evitar-se o abandono ${ }^{5}$.

Em relação ao tema discutido os autores Marcinko e Bilic ${ }^{21}$, corroboram com seus estudos ao descreverem a função protetora da psicoterapia familiar em adolescentes do sexo feminino associados com outra comorbidade, nesse estudo, especificamente foi relacionado ao transtorno alimentar. Outra pesquisa aponta que o transtorno de personalidade identificado no período da adolescência, sugere fatores de comportamentos agressivos e comportamento suicida, indicando assim, o alto índice de fatores de risco, para as características de personalidade Borderline ${ }^{14}$

Quanto aos aspectos avaliados em relação ao núcleo familiar dos adolescentes com TPB, pode-se verificar que o comportamento positivo saudável das mães desses adolescentes, evidencia envolvimento de comunicação de escuta satisfatória, auxiliando-os na diminuição dos escores considerados graves do $\mathrm{TPB}^{19}$. Em outra pesquisa, foi realizado um estudo longitudinal com o objetivo de entender a relação da agressividade parental com o TPB, a pesquisa iniciou-se, com crianças pré-escolares até a sua adolescência. Os resultados apontaram que a figura do pai com o exercício da sua autoridade, indicava características que o autor denominou de fator de proteção contra 
agressividade sintoma evidenciado no $\mathrm{TPB}^{18}$. Da mesma forma, outros autores ${ }^{17}$ corroboram a relação das famílias com problemas de relacionamentos, intrinsecamente ligado aos conflitos nas relações interpessoais, salientam que adolescentes com faixa etária ente 11 anos pode evidenciar ou apresentar sintomas descritos no DSM-IV. Além disso, as relações emocionais e afetivas desenvolvidas entre pais e filhos devem ser exploradas e analisadas, pois, pesquisas alertam sobre a importância da auto regulação emocional desenvolvidas nos adolescentes, sobretudo, nos achados com as adolescentes com sintomas de TPB ${ }^{20}$.

Dessa forma, pode-se aferir que o gênero, a estrutura familiar e o status social são elementos constitutivos, que podem afetar o desenvolvimento do transtorno de personalidade 14 .

Pode-se constatar que não é evidenciado com exclusividade o conceito dos vínculos afetivos entre pais e filhos. São descritas ações envolvendo a família, com enfoque para a psicoterapia individual dos pais e a familiar ${ }^{21,22}$. Constatou-se também a importância e o desenvolvimento do apego emocional positivo nas relações ente pais e filhos com TPB, Além disso, o comportamento afetivo materno é um fator protetor no contexto preventivo e na intervenção do transtorno ${ }^{16,19}$.

Pesquisas apontaram, a necessidades dos cuidadores serem auxiliados e amparados, no processo de tratamento da saúde mental dos membros familiares com TPB dos cuidadores, exaltando que esse grupo necessita de apoio e orientação especializada, visto que há fatores emocionais que causam prejuízo na relação interpessoal ${ }^{22}$. Os pais são vistos como figuras determinantes para um resultado positivo para o tratamento clínico e farmacológico de seus filhos 5 .

A presente revisão integrativa, acerca dos fatores associados aos pais no tratamento de adolescentes com transtorno de personalidade Borderline, ressalta a importância da presença dos pais e do vinculo afetivo positivo e saudável, durante $o$ tratamento farmacológico e terapêutico. Verificaram-se nas pesquisas encontradas, os diversos enfoques para o tratamento: hospitalar, ambulatorial, relação de apego e terapêutico ${ }^{5,14,16,19,21}$.

No que se referem à eficácia do tratamento do adolescente, as intervenções incluem além da medicação farmacológica, a terapia individual, visto que esses adolescentes necessitam verbalizar seus sentimentos frente aos problemas emocionais derivados do $\mathrm{TPB}^{21}$. Da mesma forma, o uso de intervenções positivas na regulação emocional, esta associado ao apego de segurança com as figuras parentais. Assim, ocorrendo essa regulação o adolescente se sente mais amparado para lhe dar com as suas angustias ${ }^{16}$. Visto que há estudos apontando que adolescentes com TPB sugerem um comportamento de risco elevado para cometerem suicídio ${ }^{14}$.

Análises de pesquisas apontaram que adolescentes com sintomas depressivos estavam significativamente mais comprometidos sintomatologicamente com o TPB ${ }^{15}$. Outro estudo, também destacou e valorizou o papel da mãe, como fator protetor respeitável no desenvolvimento da doença, além de auxiliar no cuidado clínico. Nessa perspectiva, os adolescentes devem ser preparados pelos médicos para 0 inicio do tratamento ${ }^{5}$, considerando-se esse processo de adequação e compreensão das peculiares a problemática vivida.

Foi percebido que a composição dos estudos avaliados, manifesta a produção de um conhecimento científico que evidencia a posição dos pais e da família frente ao tratamento de seus filhos adolescentes com TPB 14, 15, 19, 21, 22,23. Adicionalmente, a uma amplitude social descrita numa construção triangular entre os pais, filhos e tratamento.

No tocante aos pais e a família, estudos realizados corroboram e se destacam como peças fundamentais, para a obtenção de intervenções bem sucedidas ${ }^{15,}$ 16,22. As figuras parentais estiveram interligadas a aspectos emocionais construídos na relação afetiva e socioculturais. Dentre esses, encontramos que as intervenções assistidas como a terapia familiar, repercutem de forma positiva, facilitando a compreensão do tratamento ${ }^{19,}{ }^{21}$. Outro fator importante a ser considerado pelos autores ${ }^{5}$ implica ao planejamento de intervenções que envolvem o tratamento. Os pesquisadores acreditam que esse fator, seja indispensável, a fim de evitar o abandono ao tratamento do serviço médico, ou seja, é necessário um olhar especial para esses familiares do adolescente, esses devem estar esclarecidos e motivados, visando criar uma relação de comprometimento e responsabilidade com seus filhos, especialmente, durante o período de tratamento levando a intervenções satisfatórias.

Atentar sobre o abandono do tratamento clínico, constatou-se, a necessidade da elucidação das informações sobre o transtorno, explicando o planejamento a ser seguido sobre as intervenções clinicas para esses pais, a fim de 
desmitificar possíveis conhecimentos ludibriados sobre a patologia ${ }^{15}$.

Os fatores acima foram explorados, denunciando a necessidade para uma reflexão imediata, por parte dos profissionais da área de saúde (médicos e enfermeiros psiquiátricos) que assistem o adolescente em seu tratamento ${ }^{5}$. Percebemos a importância da administração farmacológica ${ }^{2122}$. No entanto, o envolvimento e o direcionamento junto aos pais, geralmente considerados os cuidadores principais dos adolescentes que se encontram em atendimento ambulatorial, nos levam a considerar, conforme já relatadas em pesquisas, uma visão peculiar assinalando expressamente, a importância dos genitores frente à aderência ao tratamento realizado ${ }^{5,22}$.

Justamente, por evidenciar, às vulnerabilidades a que estão susceptíveis os pais e adolescentes no período do tratamento, essa emblemática foi investigada por Desrosiers et al. (5) os achados denotaram a necessidade de encontrar condutas e atitudes para evitar um término precoce do tratamento, assim, seria fundamental que ocorresse uma análise juntamente com os pais e os filhos ao dar inicio a intervenção terapêutica. Precisamente, para auxiliar na constatação de possíveis causas, que pudesse e favorecesse um rompimento do tratamento clínico, ou seja, a realização de um programa interventivo seria fundamental para a consolidação de uma continuidade interventiva eficaz. Os autores acima, portanto, averiguaram que tais procedimentos são fatores de caráter prático, visto que, medos e o fato de não saber como o tratamento se processará, deixam o adolescente vulnerável. Assim sugere-se que seja explicado todo o procedimento e a forma da condução do procedimento clínico.

Foi percebido quanto aos aspectos emocionais dos pais com filhos com TPB, que eles necessitam de acompanhamento terapêutico, pois, segundo os estudos, o sofrimento da família necessita ser validados e considerados, pois, os aspectos interpessoais causados na relação pais e filhos, transfiguram-se para relações conjugais além de manifestações de adoecimento. Além disso, a família e o ambiente exerce um papel importante no comportamento dos filhos, elucubra-se que influenciá-los, sugere que a terapia familiar, possa agir de forma protetiva em torno da patologia envolvida ${ }^{22}$.

Os achados dessa revisão integrativa evidenciam que os pais necessitam utilizar-se de recursos terapêuticos, para estruturar-se emocionalmente, a fim de, colaborar com tratamento do filho, caso isso, não suceda, a pesquisa apontou que os filhos podem demonstrar um comportamento patológico comprometendo e acelerando uma desestrutura emocional ${ }^{21,22}$.

\section{Conclusões}

Os conhecimentos advindos da investigação sobre as demandas, que emergiram na pesquisa dessa revisão, apontam o tratamento e os aspectos sociais e parentais associados aos adolescentes com TPB, fundamentais para ampliar a percepção dos cuidadores responsáveis, na tarefa de auxiliar no tratamento ambulatorial recebido pelo adolescente. Percebemos que os vínculos afetivos podem ser considerados, fatores indispensáveis para que não ocorra, o abandono ao tratamento, ou mesmo, a negligência dos pais e, sobretudo, os cuidados ambulatoriais e clínicos possam ser seguidos de forma contínua, prevalecendo à alta adesão.

Ainda são incipientes publicações que analisam o vínculo afetivo desses pais com seus filhos, ou seja, que estudam o significado emocional atribuído aos pais com filhos adolescentes borderlaine em tratamento. Assim sendo, é imprescindível que ampliem o campo de conhecimento, em especial pela figura materna. Pois, a genitora exerce função significativa na saúde mental do filho. A respeito dos significados de vida atribuídos pelas mães de adolescentes com TPB, considerando que essa patologia tem um impacto considerável sobre a família e por isso, esse sofrimento deve ser validado.

\section{Referências}

1. American Psychiatry Association. Diagnostic and Statistical Manual of Mental disorders -DSM-5. 5th.ed. Washington: American Psychiatric Association, 2013.

2. Tanesi PHV, Yazigi L. Fiore, MLDM, Pitta JCDN. Adesão ao tratamento clínico no transtorno de personalidade borderline. Estudos de Psicologia. 2007; 12(1): 71-8.

3. Jordão $A B$, Ramires VR. Vínculos afetivos de adolescentes borderline e seus pais. Psicologia:Teoria e Pesquisa. 2010, 26(1): 89-98.

4. Matioli MR, Rovani ÉA, Noce MA. O Transtorno de Personalidade Borderline a partir da Visão de Psicólogas com Formação em Psicanálise. Saúde \& Transformação Social. 2014; 5(1): 50-57.

5. Desrosiers L, Saint-Jean M, Breton JJ. Treatment planning: A key milestone to prevent treatment dropout in adolescents with 
borderline personality disorder.Psichol Psychotherapy. 2015; 88(2): 178-96.

6. Mota $C P$, Rocha $M$. Adolescência e jovem adultícia: Crescimento pessoal, separaçãoindividuação e o jogo das relações. Psicologia: Teoria e Pesquisa. 2012; 28(3): 357-366.

7. Sansone RA, Farukhi S, Wiederman MW. Perceptions of childlood caretakers and borderline personality symptomatology. Child Abuse \& neglect. 2013; 37(11): 1030-3.

$8 . \quad$ Lyons-Ruth K, Bureau JF, Holmes B, Easterbrook's A, Brooks NH. Borderline symptoms and suicidality/self-injury in late adolescence: prospectively observed relationship correlates in infancy and childhood. Psychiatry Res. 2013; 30;206 (2-3):273-81.

9. Bailey RC, Grenyer BF.Supporting a person with personality disorder: a study of carer burden and well-being. Journal of personality disorders. 2014; 28(6): 796-809.

10. Wöckel L, Goth K, Zepf FD, Matic N, Holtmann M, Herpertz-Dahlmann B, Poustka F. Psychopharmacotherapy improves psychosocial functioning in adolescents with borderline personality disorder. Prax Kinderpsychol Kinderpsychiatr. 2010; 59(4): 282-301.

11. Whittemore R, Knafl K. (2005). The integrative review: updated methodology. Journal of advanced nursing. 2005; 52(5): 546-553.

12. Sobral FR, Campos CJGomes. Utilização de metodologia ativa no ensino e assistência de enfermagem na produção nacional: revisão integrativa. Revista da Escola de Enfermagem da USP. 2012; 46(1): 208-218.

13. Nunes MDR, Silva MCM, Rocha EL, Lima RAG, Nascimento LC. Mensuração de fadiga em crianças e adolescentes com câncer: revisão integrativa. Texto \& Contexto Enfermagem. 2014; 23(2): 492-501.

14. Wang $Y$, Zhu $X$, Cai L, Wang $Q$; Wang $M$, Yi J, Yao S. Screening cluster A and cluster B personality disorders in Chinese high school students. BMC Psychiatry. 2013; 13(1): 116.

15. Santisteban DA, Mena MP, Muir JMC, Brian E, Abalo C, Cummings AM. The efficacy of two adolescent substance abuse treatments and the impact of comorbid depression: Results of a small randomized controlled trial.Psychiatric Rehabilitation Journal. 2015; 38(1): 55-64.

16. Kim S, Sharp C; Carbone C.The protective role of attachment security for adolescent borderline personality disorder features via enhanced positive emotion regulation strategies. Personality Disorders: Theory, Research, and Treatment. 2014; 5(2): 125-136.
17. Wisper C, Zanarini M, Wolke D. Prospective study of family adversity and maladaptive parenting in childhood and borderline personality disorder symptoms in a non-clinical population at 11 years. Psychol Med. 2012; 42(11): 2405-20.

18. Nelson DA, Coyne SM, Swanson $\mathrm{SM}$, Hart $\mathrm{CH}$, Olsen JA. Parenting, relational aggression, and borderline personality features: associations over time in a Russian longitudinal sample. Psychological medicine. 2014; 6(3): 77387.

19. Whalen DJ, Scott LN, Jakubowski KP, McMakin DL, Hipwell AE, Silk JS, Stepp SD. Affective behavior during mother-daughter conflict and borderline personality disorder severity across adolescence. Personal Disord. 2014; 5(1): 88-96.

20. Hallquist MN, Hipwell AE, Stepp SD. Poor self-control and harsh punishment in childhood prospectively predict borderline personality symptoms in adolescent girls. J Abnorm Psychol. 2015; 124(3):549-64.

21. Marcinko D, Bilic V. Family therapy as addition to individual therapy and psychopharmacotherapy in late adolescent female patients suffering from borderline personality disorder with comorbidity and positive suicidal history. Psychiatr Danub. 2010; 22(2): 257-60.

22. Larrivée MP. Borderline personality disorder in adolescents: the He-who-must-notbe-named of psychiatry. Dialogues Clin Neurosci. 2013; 15(2): 171-9.

23. Infurna MR, Brunner R, Holz B. Parzer P, Giannone F, Reichl C, Kaess M. The Specific Role of Childhood Abuse, Parental Bonding, and Family Functioning in Female Adolescents With Borderline Personality Disorder. Journal of personality disorders. 2015: 1-16.

24. Bardin L. Análise de Conteúdo. São Paulo. Edições 70; 2011.

\section{Endereço para Correspondência}

Universidade Estadual de Campinas - UNICAMP

Cidade Universitária Zeferino Vaz - Barão Geraldo, Campinas - SP

CEP.: $13083-970$

e-mail: gerusa.abreulima@gmail.com

Recebido em 21/11/2017

Aprovado em 11/05/2018

Publicado em 30/06/2018 